\title{
Validation of Superiority of p40 over p63 in Differentiating Squamous Cell Carcinoma and Adenocarcinoma Lung
}

\begin{abstract}
Context: In this era of targeted therapy, it is important to distinguish the various subtypes of nonsmall cell lung carcinoma (NSCC). Diagnosis based on morphology alone is challenging in poorly differentiated carcinomas and core biopsies. Immunohistochemistry (IHC) helps in specifying the lineage for the subtype of NSCC. Till date, p63 is the most frequently used and sensitive marker for squamous cell carcinoma (SQCC). However, it is not specific and stains a subset of adenocarcinoma (ADC). Thus, a more reliable and specific marker is required for the diagnosis of SQCC. Objective: The objective of the study was to validate the diagnostic utility of p40 over p63 in differentiating pulmonary SQCC from ADC and NSCC-not otherwise specified (NOS). Materials and Methods: A total of 123 cases of NSCC were initially reviewed and subtyped blinded to the results of IHC. This was followed by a review of IHC slides which included p63, p40, thyroid transcription factor 1, Napsin-A, cytokeratin (CK) 5/6, and CK7. Results: There were 64 ADC, 19 SQCC, and 40 NSCC-NOS. IHC helped to confirm the morphological diagnosis in 62/64 ADCs and19/19 SQCCs. IHC classified the cases of NSCC-NOS into NSCC favoring ADC - 12 cases, NSCC favoring SQCC - 10 cases, and NSCC favoring AD-SQCC - 4 cases. Both p63 and p40 showed near equal sensitivity for SQCC (100\% and 97\%, respectively), whereas p63 showed far lower specificity when compared to $\mathrm{p} 40(51.3 \%$ vs. $100 \%)$. Conclusion: The present study confirms and validates that $\mathrm{p} 40$ is equally sensitive but highly specific than p63 in detecting SQCC. Hence, we recommend the routine use of p40 instead of p63 for the definite categorization of NSCC of the lung.
\end{abstract}

Keywords: Immunohistochemistry, lung, nonsmall cell carcinoma, p40, p63

\section{Introduction}

In the past, the only important distinction necessary was between small cell lung carcinoma (SCLC) and non-SCLC (NSCC). As targeted therapies have evolved for various subtypes of NSCC, there is an ever-increasing need for differentiating these subtypes. Distinguishing adenocarcinoma (ADC) and squamous cell carcinoma (SQCC) is important as the treatment protocols differ.

To a large extent, the hematoxylin and eosin (H and E)-stained sections are enough to study the morphology for a definite diagnosis. However, in poorly differentiated carcinomas, and more importantly for small/core biopsies, difficulty arises in subtyping NSCC accurately. It is mandatory for the small/core biopsies to be preserved for detecting the driver mutations making the tissue available for

\footnotetext{
This is an open access journal, and articles are distributed under the terms of the Creative Commons Attribution-NonCommercial-ShareAlike 4.0 License, which allows others to remix, tweak, and build upon the work non-commercially, as long as appropriate credit is given and the new creations are licensed under the identical terms.
}

For reprints contact: WKHLRPMedknow_reprints@wolterskluwer.com any required ancillary tests, especially immunohistochemistry (IHC) even less.

There are various IHC markers available to subtype NSCC. For ADC, the more important ones are the nuclear marker TTF-1 and the cytoplasmic marker, Napsin-A. SQCC has sensitive antibody markers like the nuclear marker, p63, and the cytoplasmic and membrane staining cytokeratin 5/6 (CK 5/6) antibody, among others. Novel antibodies always take antibody detection to new levels by being more specific and/or sensitive.

Till date for SQCC, anti-p63 antibody is the most frequently used nuclear marker. Although it has good sensitivity, it is not highly specific as it also stains other NSCCs. It is not useful, especially in cases of poorly differentiated NSCC-not otherwise specified (NOS) as it stains $15 \%-65 \%$ of ADC.$^{[1]}$ Hence, there is a need for a reliable, easy to assess, and a more specific marker in diagnosing SQCC.

How to cite this article: Thamtam VK, Uppin SG,
Hui M, Kumar NN, Bhaskar K, Bala SC, etal. Validation
of superiority of p40 over p63 in differentiating
squamous cell carcinoma and adenocarcinoma lung.
Indian J Med Paediatr Oncol 2020;41:535-42.

\author{
Vamshi Krishna \\ Thamtam ${ }^{1}$, \\ Shantveer G Uppin 1 , \\ Monalisa Hui ${ }^{1}$, \\ N Narendra Kumar ${ }^{2}$, \\ K Bhaskar ${ }^{2}$, \\ Stalin Chowdary \\ Bala $^{3}$, \\ G Sadashivudu ${ }^{3}$, \\ G K Paramjyothi ${ }^{2}$
}

${ }^{1}$ Department of Pathology, Nizam's Institute of Medical Sciences, Hyderabad, Telangana, India, ${ }^{2}$ Department of Pulmonology, Nizam's Institute of Medical Sciences, Hyderabad, Telangana, India, ${ }^{3}$ Department of Medical oncology, Nizam's Institute of Medical Sciences, Hyderabad, Telangana, India

Submitted: 26-Feb-2019 Revised: 25-Jun-2020 Accepted: 29-Jun-2020 Published: 29-Aug-2020

Address for correspondence: Dr. Shantveer G Uppin, Department of Pathology, Nizam's Institute of Medical Sciences, Punjagutta, Hyderabad - 500 082, Telangana, India.

E-mail:drsguppin@yahoo.co.in

Access this article online

Website: www.ijmpo.org

DOI: 10.4103/ijmpo.ijmpo_51_19 Quick Response Code:

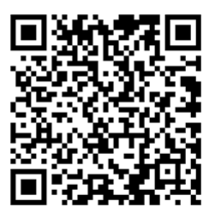


The anti-p40 antibody is highly specific for squamous/ basal cells. Studies have shown this marker to have similar sensitivity as p63 and markedly high specificity for SQCC, as it stains a lesser number of ADC. The potential pitfall of diagnosing a p63-positive ADC as SQCC can be averted to a large extent if p40 is used. In this study, we have validated the utility of p40 over p63 in differentiating pulmonary SQCC from ADC and NSCC-NOS in a large cohort of biopsies and resected specimens (RSs).

\section{Materials and Methods}

A total number of 123 consecutive cases of NSCC diagnosed on core-needle biopsies (CNBs), endobronchial biopsies (EBBs), and RSs of the lung sent for histopathological evaluation to the department of pathology between December 2014 and May 2016 were analyzed prospectively. This study was approved by the Institutional Ethics Committee. The demographic data, clinical details, radiological features, and laboratory investigations were retrieved from the medical records. The SCLCs, carcinoids, lymphomas, mesenchymal neoplasms, metastatic carcinomas, and mesotheliomas were excluded from the study.

\section{Morphological analysis}

Initially, all the cases of NSCC were morphologically categorized based on the review of $\mathrm{H}$ and $\mathrm{E}$ slides blinded to the results of special stains and IHC results. Subsequently, special stains with Alcian-periodic acidSchiff for mucin were reviewed wherever performed.

\section{Immunohistochemistry}

In all cases where IHC was done, the slides were reviewed to subtype the NSCC. In those cases where IHC was not done, the initial morphological diagnosis was considered as a final diagnosis. The primary panel of SQCC and ADC markers included p40, p63, CK5/6, TTF1, Napsin-A, and CK7. All IHCs were performed on fully automated immunostainer (Xmatrx Elite; BioGenex) by Poly horse raddish peroxide (Poly HRP) technique. Of the primary antibodies used, Napsin-A was supplied by Biocare, $\mathrm{p} 40$ by Pathinsitu, and the rest by BioGenex. All were monoclonal antibodies except $\mathrm{p} 40$.

All cases of NSCC were categorized as per the proposed International Association for the Study of Lung Cancer American Thoracic Society European Respiratory Society classification for small biopsies. ${ }^{[2,3]}$ For all the markers, the intensity of staining was taken into consideration and was compared to positive controls. For the SQCC markers, p40 and p63, nuclear staining was accepted, and cytoplasmic staining was ignored. For p40 and p63 antibody, the intensity of staining was scored semi-quantitatively using a 3-tier system: weakly positive (Grade 1), moderate positivity (Grade 2), and strongly positive (Grade 3). The staining proportion pattern was scored on a 4-tier system:
$<5 \% ; 6 \%-25 \% ; 26 \%-50 \%$; and $>50 \%$. Cases showing positivity of $5 \%$ or less were considered negative. However, $\mathrm{H}$-score was not determined.

The sensitivity, specificity, positive predictive value (PPV), and negative predictive value (NPV) of both the ADC and SQCC markers were analyzed.

\section{Results}

Of the 123 patients, nearly three-fourth, 92/123 (74.8\%) were male with a male-to-female ratio of $3: 1$. The age of the patients ranged from 18 years to 84 years, with a mean age of 57.8 years among males and 51.8 among females. Nearly $94.3 \%$ of the samples received were small biopsies, with only a small fraction of $7(5.6 \%)$ cases being surgical resection specimen. Of the biopsies, majority were computed tomography-guided CNB [95/123 (77.2\%)], followed by EBB $[20 / 123(16.2 \%)]$. There was only one biopsy which was done under ultrasound guidance.

\section{Histopathologic examination}

ADC was the most common histological subtype accounting for more than $60 \%(74 / 123)$ of NSCC cases. Of these, more than $80 \%(62 / 74)$ were diagnosed on $\mathrm{H}$ and $\mathrm{E}$ slides alone with the remaining 12 cases $(16.2 \%)$ requiring IHC for subcategorization. Of the total 64 ADC cases diagnosed on $\mathrm{H}$ and $\mathrm{E}$ morphology, majority (46 [71.9\%]), were acinar type, followed by lepidic type (10 [15.6\%]), mucinous (4 [6.3\%]), papillary (3 [4.7\%]), and fetal subtype 1 (1.5\%). Most of the acinar-type ADCs showed pure acinar morphology (38 cases) with remaining showing predominantly acinar morphology in combination with other morphologies like solid pattern (4 cases) and 1 case each of papillary, cribriform, and focal micropapillary pattern. There was one case which had an additional combination of lepidic as well as micropapillary pattern. Two of the four cases which showed predominant acinar morphology in combination with solid areas were categorized as adenosquamous carcinoma (AD-SQCC) after IHC. These two cases showed expression of SQCC markers in solid areas and ADC markers in acinar areas. Of the 10 cases of lepidic subtype, 8 cases had a pure lepidic pattern and 2 cases had additional micropapillary and acinar patterns.

Although NSCC-NOS formed the second major subtype, 40/123 (32.5\%) on H and E morphology, 24 (60\%) of these were further subtyped on IHC. These included 12 cases as NSCC favoring ADC, 10 cases favoring SQCC, and the remaining 2 cases as favoring AD-SQCC. Still, $16 / 123(13 \%)$ cases could not be further subcategorized and were retained as NSCC-NOS. Of these 16 cases, IHC slides were not available in 6 cases and the rest 10 remained uncategorized even after an appropriate panel of IHC.

SQCCs accounted for $23.6 \%$ (29/123) of the cases of NSCC in the present study. Of these, 19 (65.5\%) were 
diagnosed on $\mathrm{H}$ and $\mathrm{E}$ morphology alone, while the remaining (10 [34.5\%]) required $\mathrm{IHC}$ to favor a diagnosis of SQCC. The distribution of the cases based on histological subtype before and after IHC is provided in Table 1.

\section{Immunohistochemistry}

IHC was done in $110 / 123(89.4 \%)$ cases, of which both p40 and p63 was performed in $107(86.9 \%)$ cases. The most common IHC markers performed included lung ADC markers, TTF-1 and Napsin A, and SQCC markers, p63 and p40. The other ADC marker, CK7 and the SQCC marker, $\mathrm{CK} 5 / 6$, were performed in a smaller number of cases. The results of ADC and SQCC markers done in the NSCC cases are provided in Tables 2 and 3, respectively. The morphological and immunohistochemical features of SQCC and ADC are depicted in Figures 1-3.

Squamous cell carcinoma markers

As stated earlier, SQCC markers, p63 and p40, which were the main subjects of the present study, were performed in $107 / 123(86.9 \%)$ NSCC cases, which included both morphologically differentiated and undifferentiated NSCC cases. Both p63 and p40 showed near equal sensitivity for SQCC (100\% and 97\%, respectively), whereas p63 showed far lower specificity when compared to p40 $(51.3 \%$ vs. $100 \%$ ). Only one case in which p40 was negative but p63 was positive was a case of NSCC-NOS on initial morphology which was later categorized as NSCC favoring SQCC after IHC. This case showed additional positivity for CK5/6 and was negative for both TTF-1 and Napsin-A. None of the ADC cases were positive for p40, whereas p63 was positive in half of the cases of ADC including majority of the morphologically well-differentiated ones. Majority of ADC showed Grade 1 to Grade 2 positivity in $5 \%-50 \%$ of cells. However, there were also cases showing Grade 3 staining, as well as staining in more than $50 \%$ cells. There were five cases where p63 showed weak staining in $5 \%-25 \%$ which were not categorized as NSCC favoring SQCC and retained as NSCC-NOS. These five cases were negative for $\mathrm{p} 40$ and two of these where CK5/6 was also done showed negative staining. In one of these cases, ADC markers were negative, while in one case, they were not performed.

Of the total 4 cases finally categorized as NSCC favoring AD-SQCC, two were morphologically ADC (predominantly acinar with focal solid areas) and the remaining two were morphologically undifferentiated (NSCC-NOS). In all these cases, there were distinct areas of staining for ADC markers (TTF-1 and Napsin-A) and SQCC markers p40 and CK5/6 (done in 1 case), but the marker p63 showed

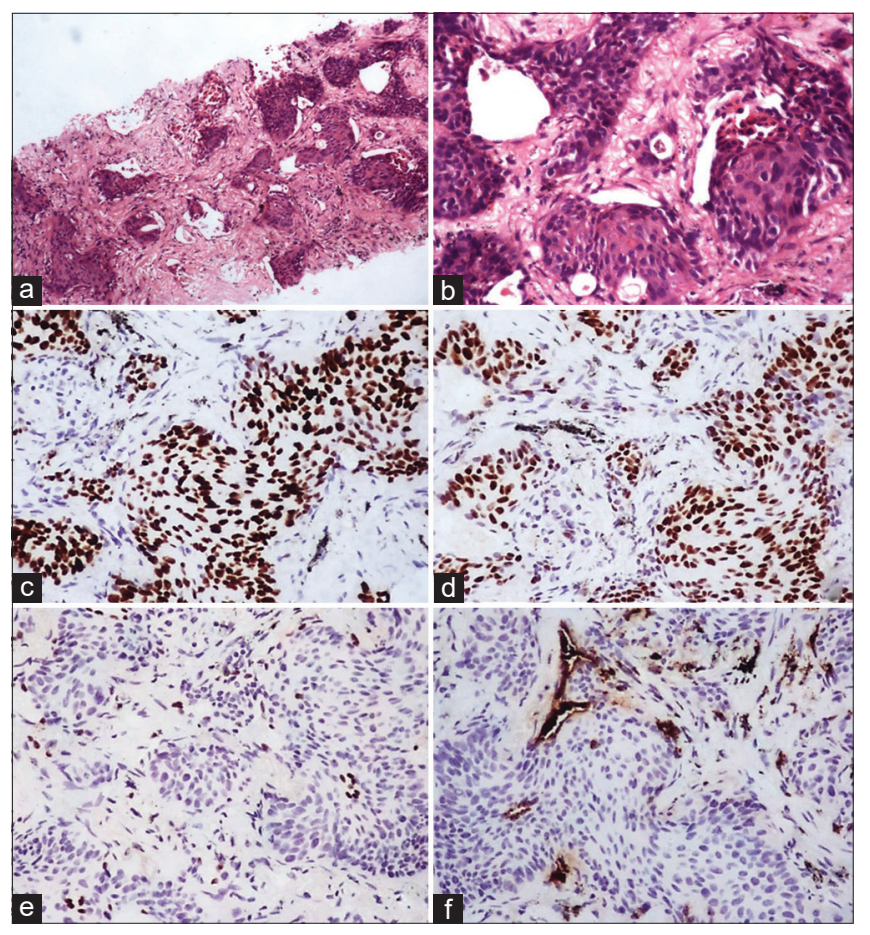

Figure 1: ( $a$ and $b)$ Squamous cell carcinoma ( $H$ and $E ; a, \times 40 ; b, \times 100)$. (c) p63 and (d) p40 showing diffuse strong nuclear staining in the tumor cells (c and d, $\times 100)$. (e) TTF-1, negative in tumor cells. Few cells showing nuclear staining at the periphery of tumor nests are normal alveolar epithelial cells which serve as internal positive control (e, ×100). (f) Napsin A, negative in tumor cells. Few cells showing granular cytoplasmic staining at the periphery of tumor nests are normal alveolar epithelial cells which serve as internal positive control $(f, \times 100)$

Table 1: Distribution of the cases of nonsmall cell lung carcinoma based on histological subtype before and after immunohistochemistry

\begin{tabular}{lllc}
\hline H and E diagnosis (before IHC) & $\boldsymbol{n}$ & Final diagnosis (after IHC*) & $\boldsymbol{n}$ \\
\hline ADC & 64 & ADC & 62 \\
& & NSCC favor AD-SQCC & 2 \\
SQCC & 19 & SQCC & 19 \\
NSCC-NOS & 40 & NSCC-NOS & 16 \\
& & NSCC favor ADC & 12 \\
& & NSCC favor SQCC & 10 \\
Total & & NSCC favor AD-SQCC & 2 \\
\hline
\end{tabular}

*IHC was not done in all cases. In such cases where IHC was not done initial morphological diagnosis was considered as final diagnosis. $\mathrm{H}$ and $\mathrm{E}$ - Hematoxylin and Eosin; IHC - Immunohistochemistry; ADC - Adenocarcinoma; SQCC - Squamous cell carcinoma; NSCC Nonsmall cell carcinoma; NOS - Not otherwise specified; AD-SQCC - Adenosquamous carcinoma 
overlapping positivity both in squamous as well as in glandular areas.

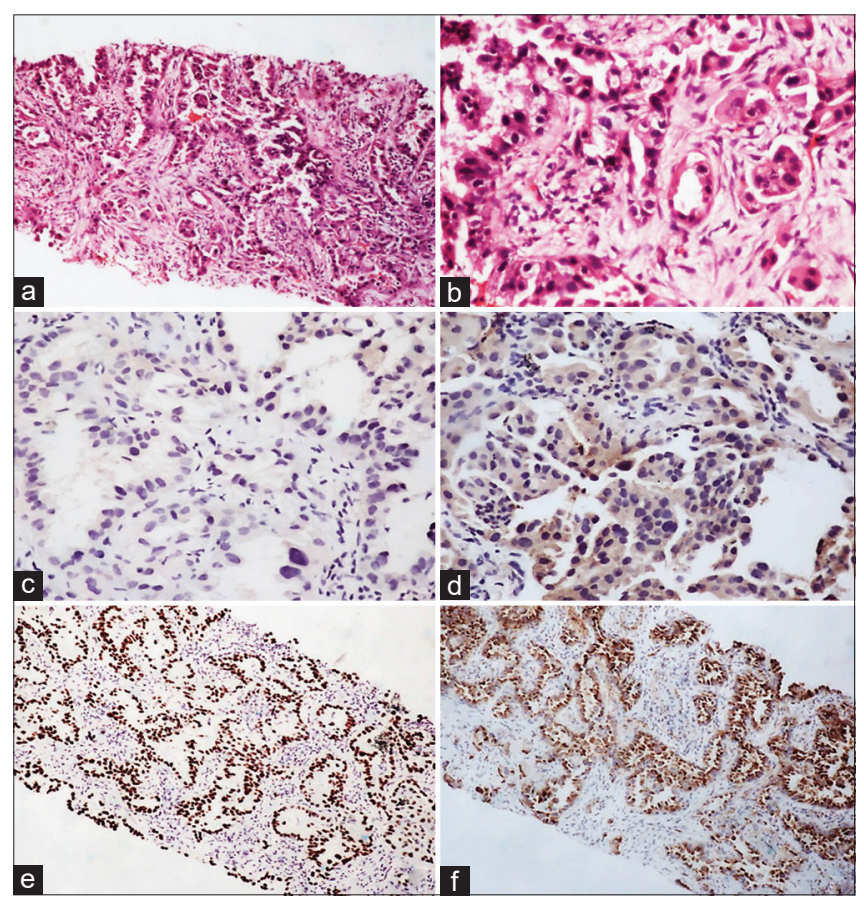

Figure 2: ( $a$ and $b)$ Adenocarcinoma showing predominantly lepidic pattern with focal acinar pattern (H and E; a, ×40; b, ×100). Both (c) p63 and (d) p40 being negative in the tumor cells. Please note nonspecific cytoplasmic staining with $\mathrm{p} 40$ (c and d, $\times 100$ ). (e) TTF-1 showing diffuse strong nuclear staining in tumor cells $(e, \times 40)$. (f) Napsin A showing diffuse strong granular cytoplasmic staining in tumor cells $(f, \times 40)$

\section{Adenocarcinoma markers}

This study also documented high sensitivities of the markers TTF-1 and Napsin-A for ADC. The sensitivity of TTF-1 and Napsin-A was $92.42 \%$ and $89.23 \%$, respectively. Although TTF-1 showed slightly higher sensitivity compared to Napsin-A, both were $100 \%$ specific. One case of invasive mucinous ADC showed negativity for both TTF-1 and Napsin-A, while for another double-negative case, no primary was found in any other part of the body even after extensive investigations; hence, it was categorized as primary lung ADC. This case was a morphologically well-differentiated ADC of acinar type and was positive for CK7.

There were four cases of ADC which showed discordant staining for Napsin-A and TTF-1. Two of cases were Napsin-A postive and TTF-1 negative, with other two showing the reverse pattern of staining. None of the ADC markers were positive in SQCC. As stated earlier, both TTF-1 and Napsin-A distinctly highlighted the ADC areas, while the remaining negative in SQCC areas of all four cases of NSCC favor AD-SQCC. Figures 4 and 5 show cases categorized as NSCC favoring ADC and SQCCC after IHC, respectively. Figure 6 depicts adenosquamous carcinoma which was initially categorized as ADC with solid and acinar patterns on morphology.

The sensitivity, specificity, PPV, and NPV of ADC and SQCC markers are provided in Tables 4 and 5, respectively.

\begin{tabular}{|c|c|c|c|c|}
\hline Final diagnosis (after IHC) & $n$ & TTF-1 (\%) & Napsin A (\%) & CK 7 (\%) \\
\hline$\overline{\mathrm{ADC}}$ & 62 & $46 / 50$ & $45 / 49$ & $14 / 14$ \\
\hline NSCC favor ADC & 12 & $11 / 12$ & $9 / 12$ & $2 / 2$ \\
\hline Total & 74 & $57 / 62(91.9)$ & $54 / 61(88.5)$ & $16 / 16(100)$ \\
\hline SQCC & 19 & $0 / 14$ & $0 / 12$ & $0 / 1$ \\
\hline NSCC favor SQCC & 10 & $0 / 12$ & $0 / 10$ & $0 / 1$ \\
\hline Total & 29 & $0 / 26(0)$ & $0 / 22(0)$ & $0 / 2(100)$ \\
\hline NSCC favor AD-SQCC & 4 & $4 / 4(100)$ & $4 / 4(100)$ & - \\
\hline NSCC-NOS & 16 & $0 / 14(0)$ & $0 / 15(0)$ & $1 / 1(100)$ \\
\hline
\end{tabular}

ADC - Adenocarcinoma; SQCC - Squamous cell carcinoma; NSCC - Nonsmall cell carcinoma; NOS - Not otherwise specified; AD-SQCC - Adenosquamous carcinoma

Table 3: Results of squamous cell carcinoma markers

\begin{tabular}{|c|c|c|c|c|}
\hline Final diagnosis (after IHC) & $n$ & P40 (\%) & P63 (\%) & CK5/6 (\%) \\
\hline$\overline{\mathrm{ADC}}$ & 62 & $0 / 56$ & $30 / 56$ & $5 / 7$ \\
\hline NSCC favor ADC & 12 & $0 / 12$ & $4 / 12$ & $3 / 12$ \\
\hline Total & 74 & $0 / 68(0)$ & $34 / 68(50.0)$ & $8 / 19(42.1)$ \\
\hline SQCC & 19 & $19 / 19$ & $19 / 19$ & $6 / 6$ \\
\hline NSCC favor SQCC & 10 & $9 / 10$ & $10 / 10$ & $1 / 4$ \\
\hline Total & 29 & $28 / 29(96.5)$ & $29 / 29(100)$ & $7 / 10(70.0)$ \\
\hline NSCC favor AD-SQCC & 4 & $4 / 4(100)$ & $4 / 4(100)$ & $1 / 1(100)$ \\
\hline NSCC-NOS & 16 & $0 / 12(0)$ & $5 / 12(41.6)$ & $1 / 4(25.0)$ \\
\hline
\end{tabular}

ADC - Adenocarcinoma; SQCC - Squamous cell carcinoma; NSCC - Nonsmall cell carcinoma; NOS - Not otherwise specified; AD-SQCC - Adenosquamous carcinoma 


\section{Discussion}

Approximately $85 \%-90 \%$ of all lung carcinomas are NSCC. ${ }^{[4]} \mathrm{ADC}$ is the largest group among NSCC, followed by SQCC, NSCC-NOS, large cell carcinoma, AD-SQCC, and sarcomatoid carcinoma. Prior to 2004, the precise subtyping of NSCC was not necessary. Treatment options for NSCC included primarily surgery, radiation, and platinum-based chemotherapy. ${ }^{[5]}$ Major therapeutic advances have had a profound impact on pathological diagnosis and molecular testing, and it is now imperative to subtype

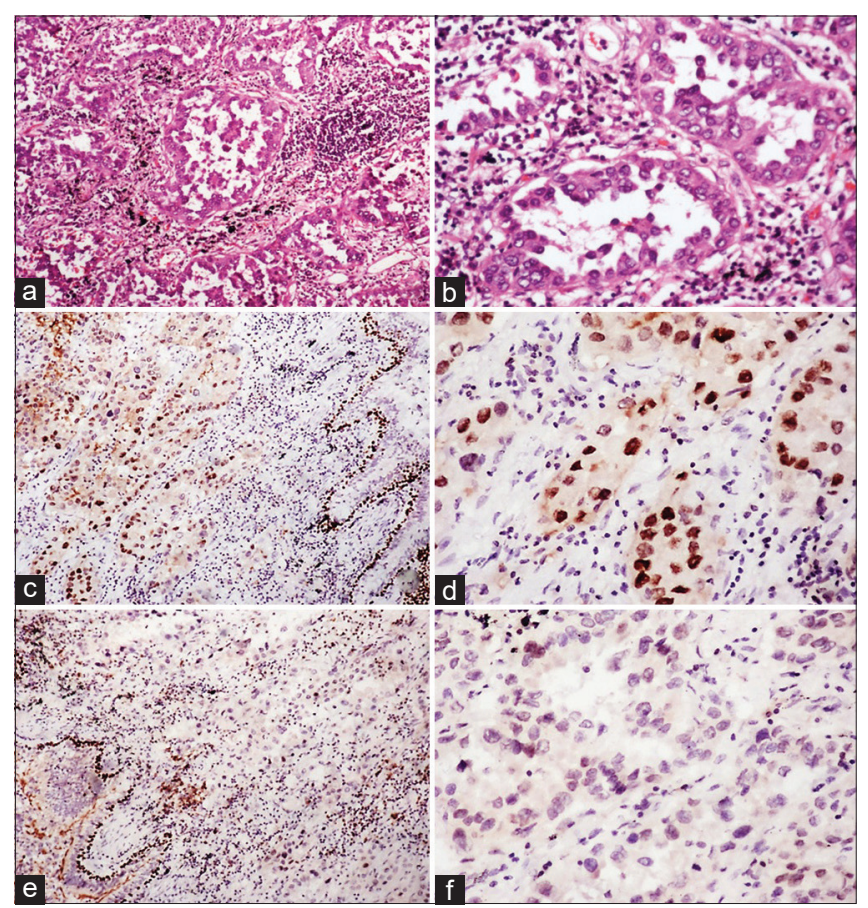

Figure 3: ( $a$ and $b$ ) Adenocarcinoma showing lepidic, acinar, and focal micropapillary patterns ( $\mathrm{H}$ and $\mathrm{E} ; \mathrm{a}, \times 40 ; \mathrm{B}, \times 100$ ). (c and d) p63 showing nuclear positivity in the tumor cells. Staining of nuclei of the basal cells of bronchiolar epithelium serves as internal positive control (c, ×40; $d, \times 100)$. (e and f) p40 being negative in tumor cells. Staining of nuclei of the basal cells of bronchiolar epithelium serves as internal positive control $(e, \times 40 ; f, \times 100)$
NSCC. ${ }^{[6]}$ Epidermal growth factor receptor mutations or anaplastic lymphoma kinase translocation in ADC have different specific targeted treatments, and so the small biopsy material needs to be conserved for molecular testing. In this context, ancillary testing, especially IHC, becomes very important in the correct diagnosis of small biopsies/cytology material and poorly differentiated lung cancers (NSCC-NOS). ${ }^{[2]}$

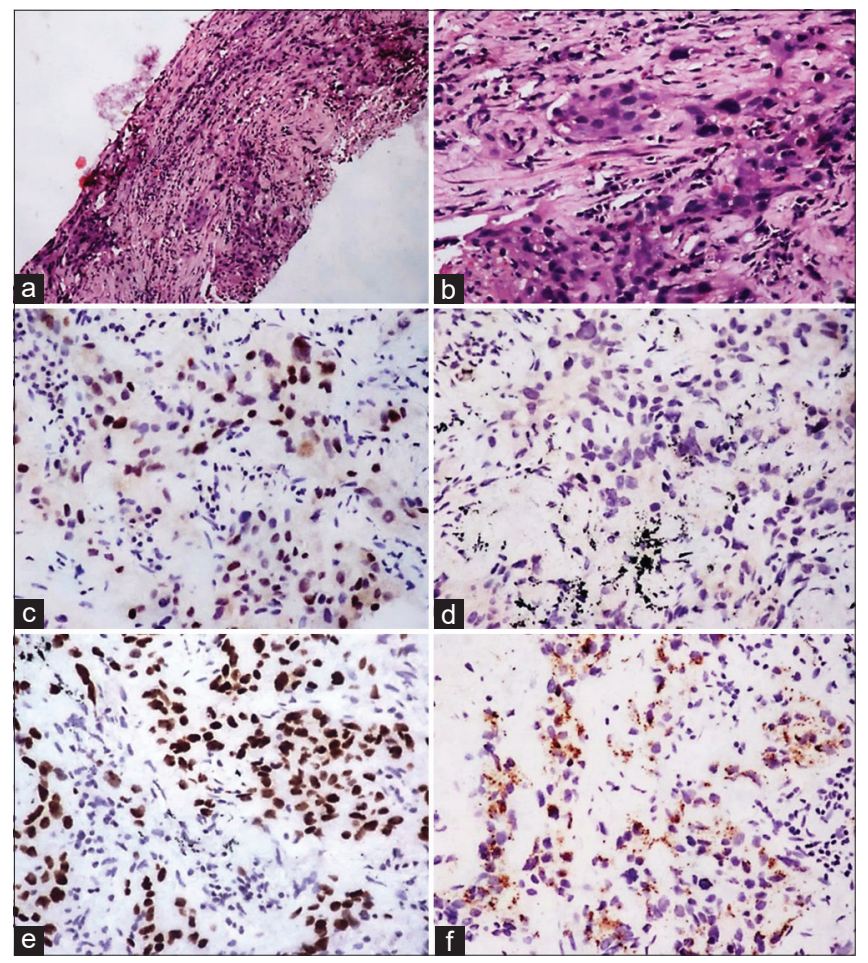

Figure 4: ( $a$ and $b$ ) Nonsmall cell lung carcinoma-not otherwise specified on morphology showing nests and cords of tumor cells infiltrating the fibrous stroma (H and E; a, $\times 40 ; b, \times 100)$. (c) p63 showing positivity within the tumor cells $(\times 100)$. (d) p40 being negative in tumor cells. (e) TTF-1 showing diffuse strong nuclear staining in tumor cells $(e \times 100)$. (f) Napsin-A showing moderate granular cytoplasmic staining in tumor cells. This case was later categorized as nonsmall cell lung carcinoma favour adenocarcinoma after immunohistochemistry

\begin{tabular}{|c|c|c|c|c|c|c|c|c|}
\hline \multirow[t]{2}{*}{ IHC marker } & \multicolumn{2}{|c|}{ Sensitivity (\%) } & \multicolumn{2}{|c|}{ Specificity (\%) } & \multicolumn{2}{|c|}{ PPV (\%) } & \multicolumn{2}{|c|}{ NPV (\%) } \\
\hline & Value & $95 \%$ CI & Value & $95 \%$ CI & Value & $95 \%$ CI & Value & $95 \%$ CI \\
\hline TTF-1 & 92.42 & $83.20-97.49$ & 100 & $91.19-100$ & 100 & $94.13-100$ & 88.89 & $75.95-96.29$ \\
\hline Napsin A & 89.23 & $79.06-95.56$ & 100 & $90.51-100$ & 100 & $93.84-100$ & 84.09 & $69.93-93.36$ \\
\hline CK7 & 100 & $79.41-100.00$ & 66.67 & $9.43-99.16$ & 94.12 & $71.31-99.85$ & 100 & $15.81-100.00$ \\
\hline
\end{tabular}

IHC - Immunohistochemistry; CI - Confidence interval; PPV - Positive predictive value; NPV - Negative predictive value

Table 5: The sensitivity, specificity, positive predictive value, and negative predictive value of squamous cell carcinoma markers

\begin{tabular}{|c|c|c|c|c|c|c|c|c|}
\hline \multirow[t]{2}{*}{ IHC marker } & \multicolumn{2}{|c|}{ Sensitivity (\%) } & \multicolumn{2}{|c|}{ Specificity (\%) } & \multicolumn{2}{|c|}{ PPV (\%) } & \multicolumn{2}{|c|}{ NPV (\%) } \\
\hline & Value & $95 \%$ CI & Value & $95 \%$ CI & Value & $95 \%$ CI & Value & $95 \% \mathrm{CI}$ \\
\hline$\overline{\mathrm{P} 63}$ & 100.00 & $89.42-100.00$ & 51.25 & $39.81-62.59$ & 45.83 & $34.02-58.00$ & 100.00 & $91.4-100.00$ \\
\hline $\mathrm{P} 40$ & 96.97 & 84.24-99.92 & 100.00 & $94.72-100.00$ & 100.00 & $89.11-100.00$ & 98.55 & 92.19-99.96 \\
\hline CK5/6 & 72.73 & $39.03-93.98$ & 60.87 & $38.54-80.29$ & 47.06 & $22.98-72.19$ & 82.35 & $56.57-96.20$ \\
\hline
\end{tabular}

IHC - Immunohistochemistry; CI - Confidence interval; PPV - Positive predictive value; NPV - Negative predictive value 


\begin{tabular}{|c|c|c|c|c|c|c|c|c|c|c|}
\hline \multirow[t]{2}{*}{ Study } & \multicolumn{5}{|c|}{ p-40 } & \multicolumn{5}{|c|}{ p63 } \\
\hline & Type of antibody & $\begin{array}{c}\text { Sensitivity } \\
(\%)\end{array}$ & $\begin{array}{c}\text { Specificity } \\
(\%)\end{array}$ & $\begin{array}{l}\text { PPV } \\
(\%)\end{array}$ & $\begin{array}{l}\text { NPV } \\
(\%)\end{array}$ & $\begin{array}{l}\text { Type of } \\
\text { antibody }\end{array}$ & $\begin{array}{c}\text { Sensitivity } \\
(\%)\end{array}$ & $\begin{array}{c}\text { Specificity } \\
(\%)\end{array}$ & $\begin{array}{l}\text { PPV } \\
(\%)\end{array}$ & $\begin{array}{l}\text { NPV } \\
(\%)\end{array}$ \\
\hline $\begin{array}{l}\text { Bishop et al., } 2012 \\
(n=470)^{[1]}\end{array}$ & Polyclonal & 100 & 98 & 92 & 100 & Monoclonal & 100 & 64 & 34 & 100 \\
\hline $\begin{array}{l}\text { Righi et al., } 2011 \\
(n=103)^{[11]}\end{array}$ & - & 100 & 96 & 94 & 100 & Monoclonal & 96 & 80 & 64 & 98 \\
\hline \multirow{2}{*}{$\begin{array}{l}\text { Tacha et al., } 2013 \\
(n=90)^{[12]}\end{array}$} & Monoclonal & 85 & 98 & - & - & Monoclonal & 85 & 90 & - & - \\
\hline & Polyclonal & 85 & 98 & - & - & & & & & \\
\hline $\begin{array}{l}\text { Collins et al., } 2013 \\
(n=100)^{[13]}\end{array}$ & Monoclonal & 89.4 & 100 & 100 & 93.9 & Monoclonal & 86.8 & 96.7 & 94.3 & 92.3 \\
\hline $\begin{array}{l}\text { Ming-Hui et al., } 2014 \\
(n=200)^{[8]}\end{array}$ & - & 80.5 & 90 & - & - & Monoclonal & 93.5 & 80 & - & - \\
\hline \multirow[t]{2}{*}{$\begin{array}{l}\text { Dvorak et al., } 2016 \\
(n=538)^{[14]}\end{array}$} & $\begin{array}{l}\text { Monoclonal } \\
\text { (BC28) }\end{array}$ & 92.6 & 94.8 & 94.1 & 93.8 & Monoclonal & 92.1 & 89.2 & 88.3 & 92.6 \\
\hline & $\begin{array}{l}\text { Monoclonal } \\
\text { (VP Echelon 40) }\end{array}$ & 75 & 98.6 & 97.9 & 81.6 & & & & & \\
\hline Present study & Polyclonal & 96.97 & 100 & 100 & 98.55 & Monoclonal & 100 & 51.25 & 45.83 & 100 \\
\hline
\end{tabular}

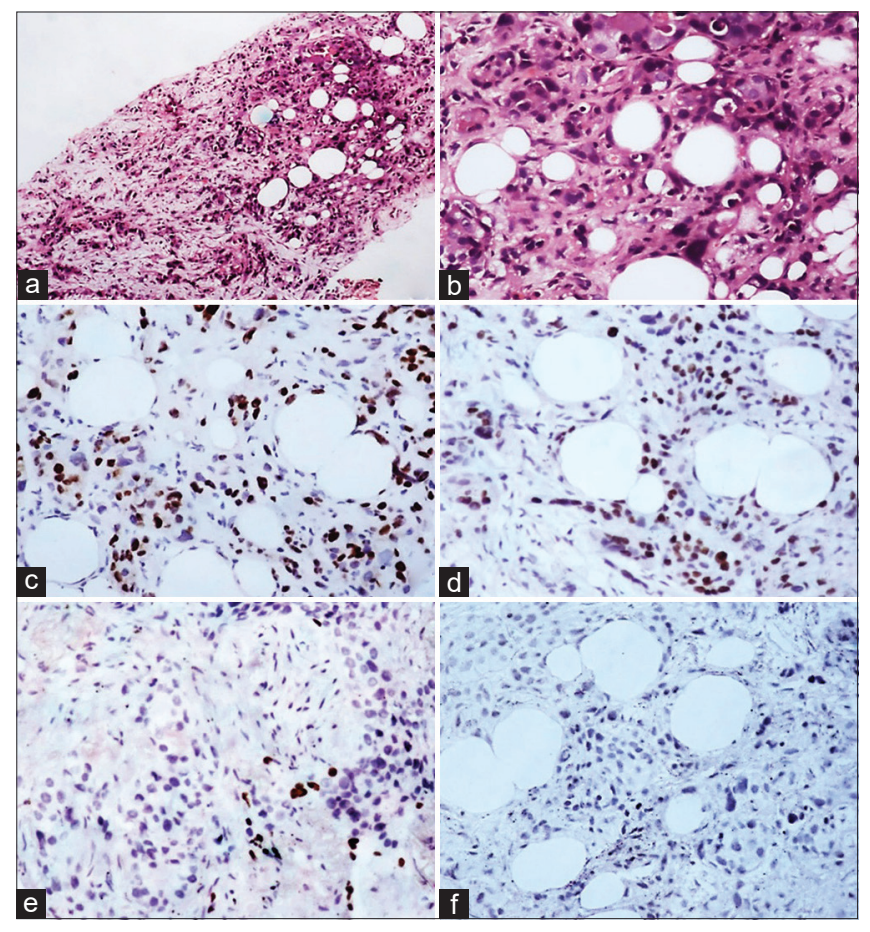

Figure 5: ( $a$ and $b$ ) Nonsmall cell lung carcinoma-not otherwise specified on morphology showing nests and cords of tumor cells infiltrating the fibrous stroma ( $\mathrm{H}$ and $\mathrm{E} ; \mathrm{a}, \times 40 ; \mathrm{b}, \times 100)$. (c) p63 and (d) p40 showing positivity within the tumor cells $(c, \times 100 ; d, \times 100)$. (d) TTF-1 and (e) Napsin-A being negative in tumor cells $(e, \times 100 ; f, \times 100)$. This case was later categorized as nonsmall cell lung carcinoma favoring SQCCC after immunohistochemistry

According to Travis et al., there is no well-established grading system for ADC, but grading according to the predominant histologic architecture is a simple and reproducible method and seems to be of prognostic importance..$^{[7]}$ In the present study, we classified ADC based on this approach. Similar to Travis et al., we also found an acinar pattern to be the most common histological pattern $(48.4 \%$ vs. $71.9 \%){ }^{[7]}$ However, unlike the present study, they found solid pattern as the second most important histologic pattern (37.7\%). There was no solid pattern in this study. The solid areas in our study were seen in combination with predominant acinar pattern in four cases. This combination of pattern probably is the reason which might have reflected a higher incidence of an acinar pattern as the predominant histologic pattern in this study.

IHC was performed in 110 of the total 123 cases in the present study, while 107 cases had both p63 and p40 performed. In the present study, it was possible to diagnose more than $80 \%$ of ADC and more than $65 \%$ of SQCC on morphology alone without the need for special stains or IHC. Among the poorly differentiated/undifferentiated cases (40/123), IHC was able to favor a definite diagnosis in 24 cases.

The antibody, anti-p63, recognizes two isoforms of the p63 gene product, the TAp63 isoform, which contains the N-terminal transactivation domain and acts as a tumor suppressor, and a truncated isoform known as p40 ( $\Delta$ Np63), which lacks the N-terminal domain and acts as an oncogene. The new anti-p40 antibody recognizes only the $\Delta$ Np63 isoform and not the TAp63 isoform. ${ }^{[1,8]}$

Many studies have established the lack of specificity of p63 for SQCC. ${ }^{[8-10]}$ Quest for a more specific antibody has brought about the novel antibody, p40, which is both sensitive and specific for lung SQCC. In the present study, the antibody p40 was nearly as sensitive as p63 for detecting SQCC but was far more specific than p63 for SQCC. According to several studies, apart from staining squamous cells, p63 also stains $15 \%-65 \%$ of ADCs. ${ }^{[1]}$ This lack of specificity is more important in the context of limited tissue availability for categorization as well as for further molecular testing to administer the targeted 


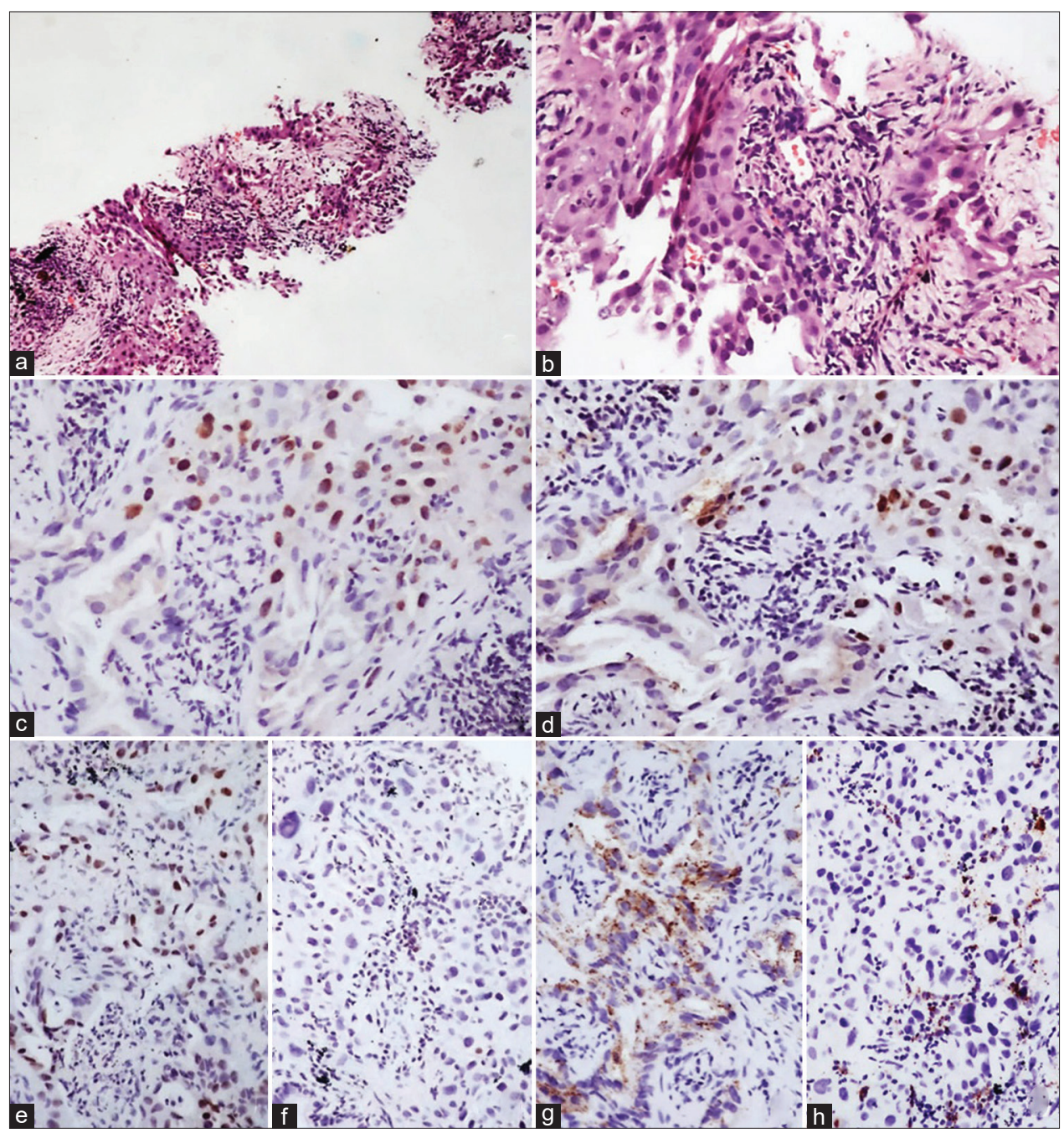

Figure 6: $(a$ and $b)$ Adenosquamous carcinoma which was initially categorized as adenocarcinoma with solid and acinar pattern on morphology $(\mathrm{H}$ and E; $\mathrm{a}, \times 40 ; \mathrm{b}, \times 100)$. (c) p63 Showing diffuse nuclear staining in solid areas. Few nuclei in the glandular areas also show positive staining. (c, $\times 100)$. (d) p40 also showed diffuse nuclear staining in solid areas but negative in glandular areas $(d, \times 100)$. (e) TTF-1 showing positive nuclear staining in glandular areas but negative in (f) solid areas $(e, \times 100 ; f, \times 100)$. (g) Napsin-A showing moderate granular cytoplasmic staining in tumor cells but is negative in $(\mathrm{h})$ solid areas $(g, \times 100 ; h, \times 100)$

therapies. In our study, p63 stained 50\% of ADC, while p40 did not stain even a single case of ADC. The comparison of IHC results for p40 and p63 in NSCC in different studies including the present series is provided in Table $6 .^{[1,8,11-14]}$

The reported sensitivity for $\mathrm{p} 40$ has ranged $75 \%-100 \%$ among various studies. The present study showed a very high sensitivity of $96.97 \%$ which is comparable to the results of Bishop et al., Righi et al., and Dvorak et al ${ }^{[1,11,14]}$ All the studies, including the present one, have shown $\mathrm{p} 40$ to be highly specific for SQCC with reported specificity ranging from $90 \%$ to $100 \%$. Our results are consistent with that reported by Collins et al. ${ }^{[13]}$ who also documented $100 \%$ specificity with $\mathrm{p} 40$. In the present study, we used a polyclonal antibody of $\mathrm{p} 40$, which has shown nonspecific cytoplasmic staining and also staining of macrophages. However, being a nuclear marker, the cytoplasmic staining was not a hindrance to the definite categorization of lung NSCC. The nuclear staining was distinctive enough to make a definite diagnosis. Bishop et al. and Tacha et al. also used polyclonal antibody for $\mathrm{p} 40$ similar to the present study and noticed similar nonspecific cytoplasmic staining. ${ }^{[1,12]}$ Tacha et al. compared monoclonal with polyclonal antibody for p40 and found no statistically significant difference in terms of sensitivity, specificity, and predictive values between the two antibodies, except for nonspecific cytoplasmic staining observed with a polyclonal antibody. ${ }^{[12]}$ Dvorak et al. compared the results of two monoclonal antibodies for p40 (BC28 and VP Echelon 40) and noticed a slightly higher sensitivity for BC28 compared to the other clone. ${ }^{[14]}$

Most of the published studies have reported p63 to be a highly sensitive antibody for SQCC with reported sensitivity ranging from $85 \%$ to $100 \%$. The present study demonstrated p63 to be $100 \%$ sensitive for SQCC, similar to that reported by Bishop et al. ${ }^{[1]}$ However, the reported specificity of p63 has varied from $64 \%$ to $97 \%$. Our study 
showed the lowest specificity of $51.3 \%$ when compared other studies. In regard to our study, the results are closer to the lower specificity of $64 \%$ reported by Bishop et al. ${ }^{[1]}$ We are unable to explain such variable specificity reported in different studies as most of the other studies used similar cutoffs as applied in the present study. In the present study, tumors positive for p63 but negative for $\mathrm{p} 40$ and CK5/6 were diagnosed as SQCC only if they were diffusely and strongly positive for p63 and negative for TTF-1 and Napsin-A.

The present study also showed TTF-1 and Napsin-A to be highly sensitive and specific markers for lung ADC. Both showed comparable sensitivities of $92.42 \%$ and $89.23 \%$, respectively, with $100 \%$ specificity. However, Tacha et al. noted slightly lower sensitivity and specificity for TTF-1 (69\% and 94.7\%) as compared to the present study. However, the sensitivity and specificity of Napsin-A were similar in both the studies. ${ }^{[12]}$

In the present study, it was possible to accurately subtype 26/40 cases of NSCC-NOS on IHC. Twelve cases favored ADC, ten cases favored SQCC, and 4 cases favored AD-SQCC after IHC. Based on our results, we recommend a panel of three markers including TTF-1, Napsin-A and p40 to subclassify most of the poorly differentiated NSCC.

\section{Conclusion}

The present study compared two lung SQCC markers - the routinely used monoclonal antibody, p63, and the novel polyclonal antibody, $\mathrm{p} 40$, and confirmed as well as validated that p40 is equally sensitive but highly specific than p63 in detecting SQCC. Despite being a very sensitive marker for SQCC, p63 lacks specificity and stains 50\% ADCs as reported in other studies. The results of the present study confirm and expand upon the previous studies that $\mathrm{p} 40$ is a more specific marker for lung SQCC than the already established marker in routine use, p63 antibody. Hence, we recommend the routine use of $\mathrm{p} 40$ instead of p63 for the definite categorization of NSCC of the lung. Our results also confirm that TTF-1and Napsin-A are equally sensitive and specific markers for ADC of the lung.

\section{Financial support and sponsorship}

Nil.

\section{Conflicts of interest}

There are no conflicts of interest.

\section{References}

1. Bishop JA, Teruya-Feldstein J, Westra WH, Pelosi G, Travis WD, Rekhtman N. p40 $(\Delta \mathrm{Np} 63)$ is superior to $\mathrm{p} 63$ for the diagnosis of pulmonary squamous cell carcinoma. Mod Pathol 2012;25:405-15.

2. Thunnissen E, Kerr KM, Herth F. The challenge of NSCC diagnosis and predictive analysis on small samples.Practical approach of a working group. LungCancer 2012;76:1-188.

3. Travis WD, Brambilla E, Noguchi M, Nicholson AG, Geisinger KR, Yatabe $\mathrm{Y}$, et al. International association for the study of lung cancer/american thoracic society/european respiratory society international multidisciplinary classification of lung adenocarcinoma. J Thorac Oncol 2011;6:244-85.

4. Siegel R, Naishadham D, Jemal A. Cancer statistics, 2012. CA Cancer J Clin 2012;62:10-29.

5. Pikor LA, Ramnarine VR, Lam S, Lam WL. Genetic alterations defining NSCC subtypes and their therapeutic implications. Lung Cancer 2013;82:179-89.

6. MitsudomiT, Morita S, Yatabe Y. Gefitinib versus cisplatin plus docetaxel in patients with nonsmall cell lung cancer harbouring mutations of the epidermal growth factor receptor (WJTOG3405): An open label, randomised phase 3 trial. Lancet Oncol 2010;11:121-28.

7. Travis WD, Rekhtman N, Riley GJ, Geisinger KR, Asamura H, Brambilla E, et al. Pathologic diagnosis of advanced lung cancer based on small biopsies and cytology: A paradigm shift. J Thorac Oncol 2010;5:411-4.

8. AO MH, Zhang H, Sakowski L, Sharma R, Illei PB, Gabrielson E, et al. The utility of a novel triple marker (combination of TTF1, napsin A, and p40) in the subclassification of non-small cell lung cancer Hum Pathol 2014;45:926-34.

9. Weinstein IB, Joe AK. Mechanisms of disease: Oncogene addiction-a rationale for molecular targeting in cancer therapy. Nat Clin Pract Oncol 2006;3:448-57.

10. Nonaka D. A study of $\Delta \mathrm{Np} 63$ expression in lung non-small cell carcinomas. Am J Surg Pathol 2012;36:895-9.

11. Righi L, Graziano P, Fornari A,Rossi G, Barbareschi M, Cavazza A, et al. Immunohistochemical subtyping of nonsmall cell lung cancer not otherwise specified in fine-needle aspiration cytology a retrospective study of 103 cases with surgical correlation. Cancer 2011;117:3416-23.

12. Tacha D, Bremer R, Haas T, Qi W. An immunohistochemical analysis of a newly developed, mouse monoclonal p40 (BC28) antibody in lung, bladder, skin, breast, prostate, and head and neck cancers. Arch Pathol Lab Med 2014;138:1358-64.

13. Collins BT, Wang JF, Bernadt CT. Utilization of p40 $(\Delta \mathrm{Np} 63)$ with p63 and cytokeratin $5 / 6$ immunohistochemistry in non-small cell lung carcinoma fine-needle aspiration biopsy. Acta Cytologica 2013;57:619-24.

14. Dvorak K, Yambert J, Palting J, Reinhardt K, Roessler C, Moh A, et al. Improved lung cancer classification using new optimized immunohistochemical assay with anti-p40 (BC28) mouse monoclonal antibody. Int J Clin Exp Pathol 2016;9:2693-701. 